\title{
Correlates of conflict resolution across cultures
}

\author{
Zachary H. Garfield ${ }^{1}$ \\ ${ }^{1}$ Institute for Advanced Study in Toulouse, Université de Toulouse 1 Capitole, Toulouse, \\ France
}

zachary.garfield@iast.fr

Preprint of manuscript resubmitted to Evolutionary Human Sciences as of 27 July 2021. Also archived at Zenodo DOI: 10.5281/zenodo.4683028.

\section{Abstract}

Conflicts are ubiquitous between individuals as well as between groups. Effective conflict resolution is essential for individual well-being and group functioning and often involves leadership dynamics. The evolutionary human sciences have suggested conflict resolution is shaped by psychological heuristics, norms, and ecology. There is limited empirical data, however, on conflict resolution across cultures. Using a cross-cultural database of 109 leadership dimensions coded from over 1,200 ethnographic texts from the eHRAF ethnographic database, exploratory analyses investigated correlates of conflict resolution. Results revealed greater evidence of conflict resolution among kin groups than political groups and greater evidence of within-group conflict resolution than between-group, which did not vary across subsistence strategies or group contexts, with two exceptions military groups conflicts were biased towards between-group contexts and religious groups biased towards within-group contexts. The strongest predictors of conflict resolution services were other prosocial functions and included group representation and providing counsel, protection, and punishment, as well as qualities of interpersonal skills and fairness. Followers received social service benefits and reduced risk of harm. For leaders who resolve conflicts, status and social benefits were potential negative predictors. These results provide a comparative view of the correlates of conflict resolution, suggesting diversity across social contexts.

\section{Introduction}

Humans are exceptional for our abilities to sustain large-scale cooperation, including between kin, co-residents, strangers, and groups (Santos and West 2018). Diverse nonhuman social species also demonstrate substantial cooperation (e.g., Allee 1951). Cooperative relationships, however, are vulnerable and can decay if conflicts between individuals or groups go unresolved. Group living increases opportunity for interindividual conflict and across mammals, conspecific killing is most frequent among species that engage in territorial defence, and among territorial primates especially (Gómez et al. 2016). Unsurprisingly, dispute settlement mechanisms are widespread across group-living species and conflict resolution is a universal feature of human sociality (Brown 1991; Aureli, Waal, and Waal 2000). 
The characteristics of individuals who resolve conflicts, the associated costs and benefits of conflict resolution, and the contexts that present greater conflict resolution demands have been widely discussed across disciplines, but empirical evidence from a representative sample of human social and cultural diversity has remained unavailable. Through exploratory analyses the present study aims to provide new cross-cultural insight into these empirical gaps.

\section{Sociality and demands of conflict resolution}

Most species, including about 70\% of mammals, do not live in groups (Wilson and Reeder 2005 ) and the evolution of group living faces significant challenges including opportunities for conflict. Individuals in close proximity can be in conflict, for example, over access to material resources, mating opportunities, and territory (Parker 2006; Smith 1985; Ross 1983). Co-residents and kin can develop additional conflicts within a social structure including over position in a status hierarchy or in economic exchange (Parker, Royle, and Hartley 2002; Hames 2015). Humans also develop coalitionary and inter-group conflicts across levels of social organization (e.g., kin, residential, or political levels) (Roscoe 2009; Glowacki, Wilson, and Wrangham 2017; Redhead and Von Rueden 2021).

Despite these challenges, for a minority of mammals including humans and most primates, group living is obligatory (Hrdy 2006). Group living then must have in some contexts offered individuals a net fitness benefit over evolutionary history (Alexander 1974). Putative benefits include reduced predation risks, coordination to accomplish highly profitable yet difficult goals, and increased abilities to control territory (Willems and van Schaik 2017; Smith et al. 2012). In the context of group living, co-residents who effectively solve inter-individual conflicts can benefit both at the individual-level via reduced aggression (direct and indirect) and increased cooperation (Alexander 1974; Chapman and Valenta 2015). Groups also stand to benefit from conflict resolution mechanisms via cultural group selection processes and mutually beneficial cooperation (Richerson et al. 2016).

\section{Leadership in conflict resolution}

Although conflicts are common, conflict resolution mechanisms are also common and often associated with a leadership role. Theoretical models suggest cooperative dynamics can emerge in conflict-prone groups in the context of inter-individual heterogeneity. For example, dominant leaders, or individuals who maintain influence via aggressive or coercive strategies (Mesterton-Gibbons and Dugatkin 1995; Cheng 2019), may more effectively enforce norms, levy punishments, and provide conflict resolution services than non or less dominant individuals (Mesterton-Gibbons et al. 2011; Redhead, Dhaliwal, and Cheng 2021). Such services, however, also present opportunities for individual costs and collective action dilemmas. Despite dominance, high status, or leadership status, thirdparty individuals who resolve conflicts individuals may face reputational costs (Raihani and Power 2021) and may face counter-punishment or aggressiveness from individuals in conflict if the mediation is perceived as unjust (Jensen 2010; Bøggild and Petersen 2016). Between-group dynamics, including competition between dominant or high ranking individuals, can also promote within-group cooperation and disproportionate group 
investment by high ranking individuals, which could include costly conflict resolution

74 services (Gavrilets and Fortunato 2014).

Evolutionary anthropologists have emphasized the role of prestigious individuals, senior kin, and other influential community members as mediators of conflicts within and between kin groups across cultures, and have suggested conflict resolution processes underlay much of biological and cultural evolution (Glowacki and von Rueden 2015; Garfield, Hubbard, and Hagen 2019; Boehm 2013). Drawing on ethnography and empirical field data from smaller-scale, politically autonomous populations Glowacki and von Rueden (2015) frame within-group conflict resolution as one type of collective action problem groups must overcome. They suggest 1 ) effective leadership emerges and is selected for due to demands of resolving collective action conflicts within institutional systems, 2) individuals with wider social networks, greater knowledge, and physical formidability will be best equipped to effectively resolve inter-individual conflicts; and 3) institutionalized leadership (i.e., culturally transmitted norms proscribing by whom and how leadership operates) likely first emerged to facilitate conflict resolution within kin groups.

Smith et al. (2016) provide a cross-species and cross-cultural comparison of leadership in within-group conflict resolution, contrasting nonhuman animal societies with small-scale human societies. Across their sample, within-group conflict resolution was not widely distributed, i.e., a few individuals had greater influence in conflict resolution in both nonhuman and small-scale human societies compared to other group members, implicating the role of individual leaders, or individuals who have disproportionate group influence (von Rueden and van Vugt 2015), in the resolution of within-group conflicts. Smith et al. (2016) also found that among both nonhuman and small-scale human societies leaders and followers generally equally benefited from within-group conflict resolution and conflict resolution roles were more often achieved than ascribed, suggesting group dynamics shape the qualities of leaders who resolve conflicts. In small-scale human societies, however, leaders who resolve within-group conflicts were more likely to have to coercive power (Smith et al. 2016). Followers then are at risk of exploitation via mediation processes (as well as decision-making hierarchies, generally) and influential individuals such as community leaders may also initiate conflict in effort to implement policies (Boehm 1999, 2008).

Given the the universality of conflict resolution across human populations and its broad phylogenetic distribution, there are likely to be evolved psychological mechanisms facilitating conflict resolution strategies, as well as facultative responses to ecological conditions and culturally evolved systems exhibiting variability and convergence. Current perspectives highlight the need for additional research on conflict resolution and leadership across cultures and contexts. Moreover, the cultural and social diversity of within-group conflict resolution is not well documented, given much of the evolutionary social science on conflict has focused on warfare and between-group conflict (e.g., Glowacki, Wilson, and Wrangham 2017; Sluka 1992; Lopez 2020). 
114 Conflict resolution occurs among every human society but we currently know very little

115

116

117

118

119

120

121

122

123

124

125

126

127

128

129

130

131

132

133

134

135

136

137

138

139

140

141

142

143

144

145

146

147

148

149

150

151

152

153

about whether such processes utilize similar or variable social mechanisms (e.g., coercion or persuasion) and if they rely on the same individual characteristics across the diversity of human social and cultural contexts (e.g., high social status, large social networks, or fairness). Addressing such questions requires a broad, comparative, and cross-cultural framework. Based on current theoretical and empirical literature, outstanding questions on human conflict resolution include: 1) what are the underlying behavioural, personality, or other characteristics that characterize leaders who resolve conflicts across diverse cultural and social contexts?; 2) what are the specific costs or benefits individuals incur from conflict resolution processes?; and 3) how widespread is coercive authority or prosociality of leaders who solve disputes, across diverse societies? Lastly, 4) does group context or cultural typologies predict variation in human conflict resolution? The present study aims to provide some insight to these questions through an exploratory analysis leveraging a novel cross-cultural database (Garfield and Hagen 2019).

Conflict resolution was the most cross-culturally frequent function of leaders, documented in $78 \%$ of cultures in a large sample of ethnography from 59 cultures (Garfield, Syme, and Hagen 2020). Given the frequency of conflict resolution in the ethnographic record and important outstanding questions, further analyses are warranted. The current study focuses in detail on the "conflict resolution" variable in the Garfield and Hagen (2019) database to identify the characteristics of leaders who resolve conflicts and the social and cultural contexts more strongly associated with conflict scenarios. The correlates of conflict resolution speak to the behavioural and phenotypic traits of individuals who resolve conflicts and to the cultural views of conflict resolution across human societies. Individual, group, and culture-level measures will be used to predict evidence for conflict resolution to identify how conflict resolution may covary with cultural and social ecology. Lastly, textanalysis is used to provide insight into the semantic content of the ethnographic record of conflict resolution.

\section{Methods}

\section{Cross-cultural database}

This study uses the leadership data package (Garfield and Hagen 2019), a recently developed cross-cultural database, designed to capture a wide range of ethnographic content related to leadership from the 60-cultural Probability Sample Files (Naroll 1967) of the electronic Human Relations Area Files (eHRAF). The eHRAF is an electronic database of primary ethnographic documents which can be queried using a thorough subject code system (Outline of Cultural Materials or OCM codes) and/or by keyword, at the paragraph level. The leadership data package is based on 1212 ethnographic paragraphs (termed text records) extracted from the eHRAF using a broad search strategy targeting general descriptions of leadership. These text records stem from from 321 documents describing 59 cultures (see Figure $\mathrm{S} 1$ for the geographic distribution of the culture sample). The data package includes researcher-coded measures of evidence for 109 dimensions of leader 
154 qualities and functions (coded as "evidence for" = 1 or "no evidence" = 0), including conflict 155 resolution (the Resolve conflict variable), as well as measures of costs and benefits for 156 leaders and followers, a measure of group context (see Table 1), the context of leader 157 functions (i.e., within-group, between-group, or both), and culture and document-level 158 metadata (e.g., subsistence strategy, year of publication, author). See Tables S1 and S2 in 159 the supplementary information for operational definitions of all leadership dimensions.

160 As example, the following text record from Pospisil (1963) on the Kapauku agriculturalists 161 of the Indonesian Central Highlands was coded as providing evidence for the functions of

162 Resolve conflict and Punishment, with the group context of kin group and the context of 163 conflict as within-group:

164 The behavior of members of a sublineage (or of a non-subdivided lineage) discloses mutual affection and a strong 165 sense of belonging and unity. Within this group all fighting is considered deplorable. Even an organized stick fight, 166 which occasionally disrupts the otherwise cordial relations within a lineage, is unheard of in this subgroup. The 167 main responsibility of the leader of the sublineage is to prevent or to quell an occasional brawl and to mete out a 168 deserved punishment in accord with customary law.

169 For additional details on database construction see Garfield, Hubbard, and Hagen (2019) 170 and Garfield, Syme, and Hagen (2020).

171 Table 1: Operationalization of the group context variable. Reproduced from Garfield et 172 al. (2020).

173

\begin{tabular}{|c|c|}
\hline Group context & Description \\
\hline Residential subgroup & $\begin{array}{l}\text { Informal groups of co-residents, social groups, age-based groups, or performance } \\
\text { groups }\end{array}$ \\
\hline Kin group & Groups based on kin relationships, such as lineages, phratries, and clans \\
\hline Economic group & $\begin{array}{l}\text { Subsistence groups, market groups, and other groups with primarily economic } \\
\text { goals }\end{array}$ \\
\hline Military group & All groups related to inter-group conflict \\
\hline Religious group & Groups formed for spiritual or supernatural purpose \\
\hline Political group (community) & $\begin{array}{l}\text { Political groups at the level of the community, i.e., political leaders, such as village } \\
\text { headmen, can potentially interact directly with most community members }\end{array}$ \\
\hline $\begin{array}{l}\text { Political group } \\
\text { (supracommunity) }\end{array}$ & $\begin{array}{l}\text { Political groups that encompass multiple residential communities, such as } \\
\text { complex chiefdoms, regional political leaders, and kings or state-level leaders }\end{array}$ \\
\hline
\end{tabular}


176 The first goal of the current study was to was to identify which of the other 108 leadership dimensions most strongly associated with evidence for conflict resolution processes. A logistic elastic net regression model was fit with all leadership dimensions as predictors of evidence for the Resolve conflict measure. Elastic net regression models are penalized regression models that are effective when the number of predictors is large relative to the number of observations. Elastic net regression models in the current study used the glmnet package (Friedman, Hastie, and Tibshirani 2010) and the "lasso" penalty $(\alpha=1)$, which will often set many coefficients to 0 , thereby identifying the most important predictors among all covariates. Following standard procedure, 10 -fold cross-validation was used to find the optimum value of the penalty term $\lambda$, i.e., the value that minimized cross-validation error. A second value of $\lambda$ was also selected that was the largest value of lambda such that the error was within 1 standard error of the minimum, i.e., one that would increase shrinkage relative to the optimal $\lambda$ and therefore decrease false positives. For the elastic net regression model of Resolve conflict (coded as 0 for no evidence, 1 for evidence for) coefficients from both the optimal $\lambda_{\text {min }}$ model and the more conservative $\lambda_{1 S E}$ model are reported. All variables were centred and standardized by one standard deviation prior to fitting. See the SI for further details and motivations on elastic net models.

The second goal was to assess social and cultural variation associated with evidence for conflict resolution, namely by subsistence strategy (see Figure S1) and group context (see Table 1). This goal involved via two methods. First, the ethnographic evidence for conflict resolution (i.e., Resolve conflict $=1$ ) was descriptively represented in relationship to group context (e.g., kin groups vs. political groups), subsistence type (e.g., hunter-gatherers vs. agriculturalits), and the type of conflict (i.e., within-group vs. between-group conflicts). Second, the Resolve conflict variable was used as an outcome in logistic mixed effects models using the lme4 package (Bates et al. 2015) with subsistence type, group context, and continental region as predictors.

The third goal was to empirically describe the ethnographic record of human conflict resolution through text analyses. Ethnography-based data are rooted in bodies of text produced by ethnographers and other authors. The semantic content of these documents can be analysed using text analytic methods to supplement analyses from researcher-coded variables. A document-term matrix was developed of all "informative" words in the corpus of text records. The "informative" words are the unique words produced by excluding stop words (e.g., "a", "the", "is", "are") and word "stemming" which removes suffixes (e.g., "quarrelling", "quarrelled", "quarrels" are all be reduced to "quarrel"). In this matrix each row corresponds to one of the 1,212 text records in the sample and each column to one of theses 9,656 unique "informative" words. Each cell value in the document-term matrix indicates the number of times that word appears in that text record. Included alongside the document-term matrix is a column for the researcher-coded Resolve conflict variable. An elastic net logistic regression model (with the lasso penalty, $\alpha=1$ ) of Resolve conflict was fit as a function of the frequencies of all 9656 words. Words that were strong positive predictors exemplify the semantic content of the text records which provided evidence for conflict resolution. 
218 All analyses were conducted with R version 4.0.2 (2020-06-22).

\section{Results}

220 The number of text records per culture in leadership data package sample ranged from 1 to 126 , with a median of 13 , a mean of 20.5 , and a standard deviation of 23.4. The text records were generally short, ranging from 14 to 1402 words, with a median word count of 141 , a mean of 159.5, and standard deviation of 97.4. Publication dates of documents in the leadership data package ranged from 1860 to 1999, with a median year of 1964. There were 217 male authors, 62 females authors, and 3 authors whose gender was not determined (some were co-authors). Of the 321 source documents, 70 (22\%) had a female author or co-author. The total pages of eHRAF ethnography on each culture in the culture sample ranged from 934 to 11,234 , with a median of 2,771 , and this was weakly correlated with the number of text records on leadership retrieved for a particular culture $(r=0.24, p$ $=0.062$ ). For additional detailed descriptive results of ethnographic paragraphs in the leadership data package see Garfield, Syme, and Hagen (2020), including analyses of potential bias by ethnographer gender, year of publication, and total pages of ethnography per culture (overall, potential biases from these measures were deemed to be negligible).

\section{Leadership dimensions predictive of conflict resolution}

An elastic net logistic regression model (with the lasso penalty, $\alpha=1$ ) of Resolve conflict was fit as a function of the other 72 quality and function leadership dimensions, and the 36 leadership costs and benefit measures (nine measures which include costs and benefits variables for both leaders and followers, i.e., $9 \times 2 \times 2$ ). This model identifies which dimensions are associated with evidence for conflict resolution in this sample of the ethnographic record of leadership.

The non-zero coefficients under the more conservative $\lambda_{1 S E}$ indicated that the functions Group representative and Provide counsel/direction were the strongest positive predictors and the functions Protection and Punishment as well as the qualities Interpersonal skills and Fairness were moderate or weak positive predictors of evidence for Resolve conflict (Figure 1). The $\lambda_{\text {min }}$ estimates also identified a suite of other qualities, functions, and benefits, including Ritual functions and Control economics and the follower benefits of Social services and Reduced risk of harm. Interestingly, two benefits for leaders, Increased social status and Social services were negative predictors under the $\lambda_{\min }$ model. 


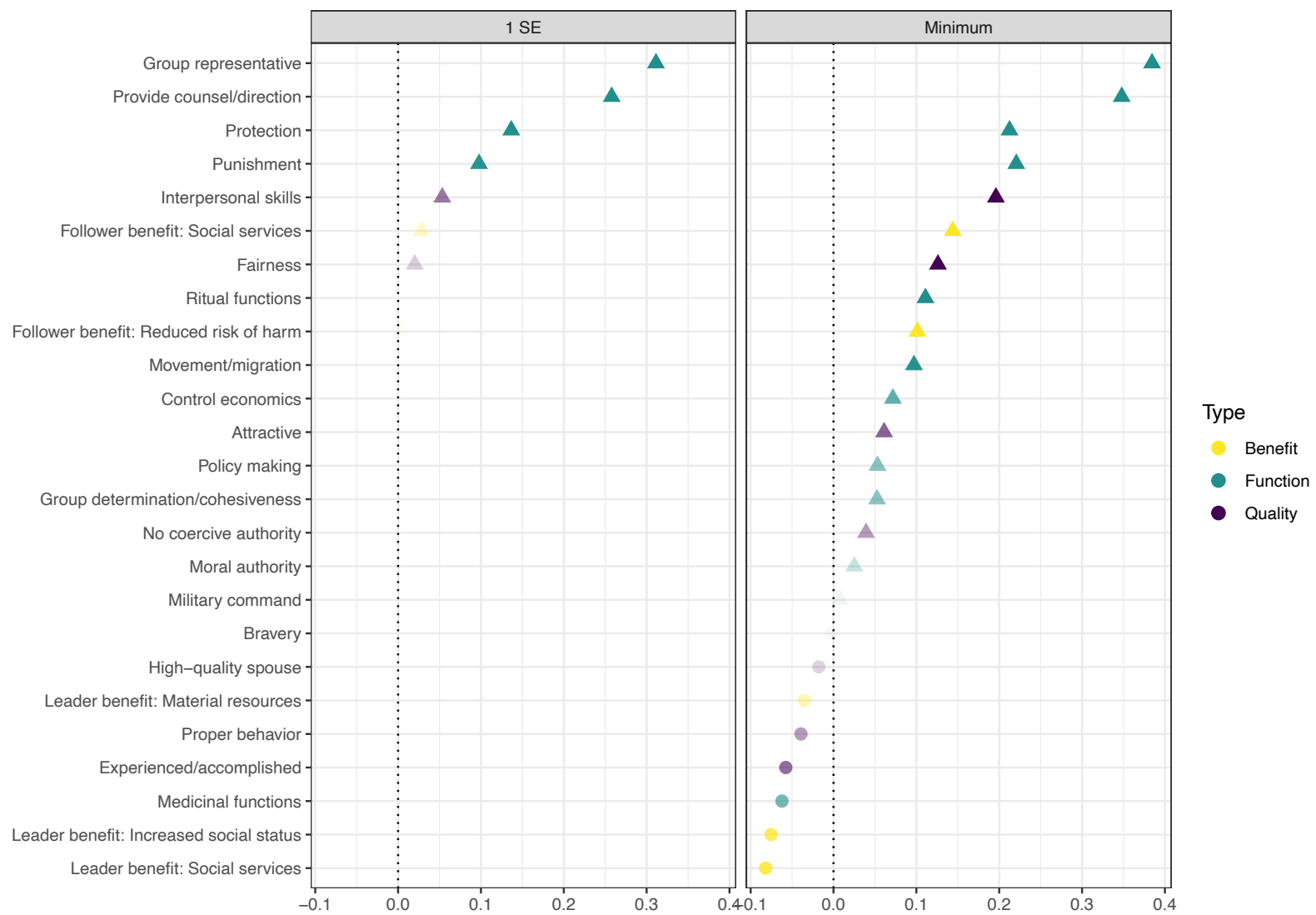

Figure 1: Non-zero coefficients of leadership dimensions that predicted evidence for Resolve conflict using the lasso penalty $(\alpha=1)$, with $\lambda_{\min }$ (value $\left.=0.01\right)$ chosen by cross-validation. Coefficients from $\lambda_{1 S E}$ (value $=0.03$ ) are those under $\lambda$ values within one standard error from $\lambda_{\text {min. }}$. Color indicates leadership dimension type (for illustration only). Shape indicates positive vs. negative predictors. Point transparency is proportional to coefficient value for low values. Predictors with coefficients $=0$ not displayed. $X$-axes are log odds on the response scale.

\section{Social and cultural variation in evidence for conflict resolution}

Descriptive results of conflict resoultion by subsistence and group context

Of the 1,212 text records, 152 provide evidence for conflict resolution. The distribution of evidence for conflict resolution across subsistence type and group context (see Table 1) and in relationship to the context of conflict (e.g., within-group, between-group) is depicted in Figure 2, for these 152 text records. The Context of conflict was coded as "within-group", "between-group", "both", or "unknown" ("unknown" was only coded for three text records and has been removed from these figures).

In the mosaic plot in Figure 2, the area of each bar is proportional to the number of text records providing evidence for Resolve conflict in that category. In Figure $2 \mathrm{~A}$ the $\mathrm{x}$-axis reveals the unbalanced evidence across subsistence types and in Figure 2B across group context. This plot also reveals that across cultures with distinct subsistence types (Figure $2 \mathrm{~A}$ ), the ethnography of conflict resolution does not substantially vary across within or 
270 between-group conflicts and there is a substantial bias in favour of evidence for withingroup conflict resolution processes, with one possible exception that pastoralists demonstrated slightly more relative evidence for between-group conflict resolution than other subsistence groups. Across all subsistence types, however, there is evidence of between-group conflict resolution processes.

Across group contexts there are also general similarities in the distribution of within and between-group conflict resolution (Figure 2B), with two obvious exceptions; leaders of religious groups primarily resolve within-group conflicts (with marginal evidence of conflicts involving both contexts) and leaders of military groups are primarily involved in between-group conflict resolution, though there is evidence of within-group conflict 280 resolution among military leaders as well.

Mosaic plots of evidence for conflict resolution

A

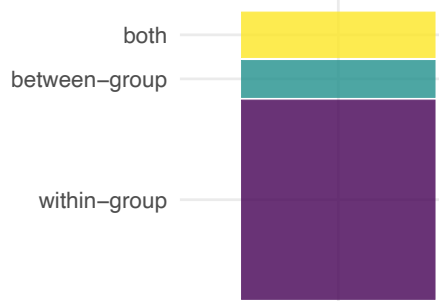

hunter gatherers

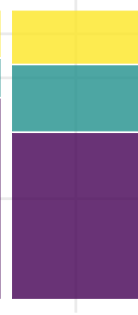

pastoralists

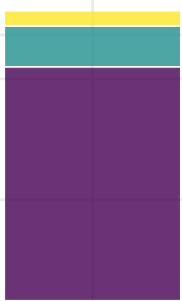

mixed

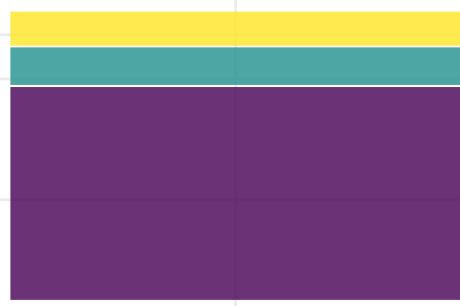

horticulturalists

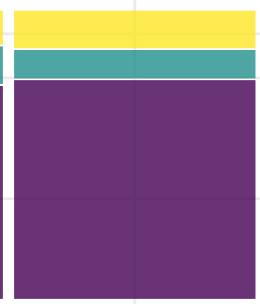

agriculturalists

B
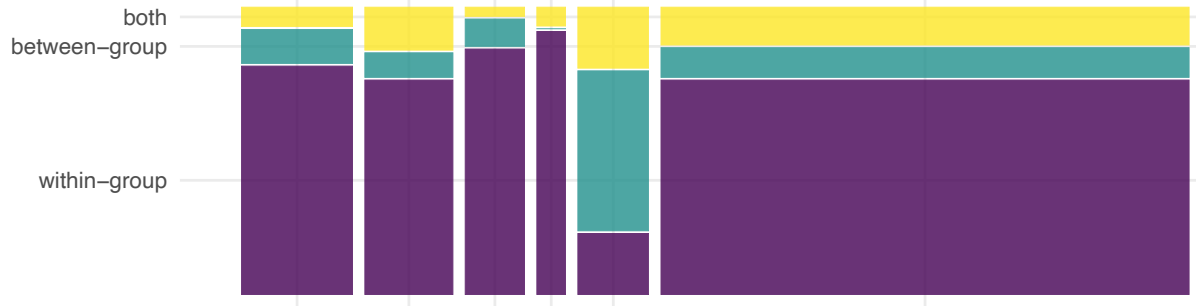

residential subgroup

political group

(community)

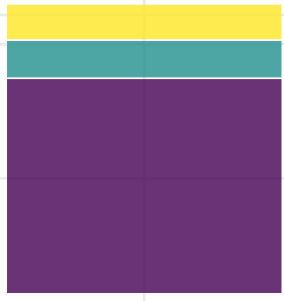

political group (supracommunity)

Figure 2: Mosaic plots of the distribution of evidence for conflict resolution. The $y$-axis and colour indicate the Context of conflict coding. A: By subsistence and the type of conflict. B: By Group context and type of conflict. Areas of the bars are proportional to the number of text records providing evidence for conflict resolution in that category.

\section{Predicting variation in conflict resolution}

287 In their systematic analyses of all 109 leadership dimensions in the leadership data package, Garfield, Syme, and Hagen (2020) identified conflict resolution as a candidate universal of human leadership (see their Table 1), given this measure did not meaningfully vary by continental region, subsistence strategy, group context, or leader sex - four predictors applied to all leadership dimensions - relative to an intercept-only model. There 
292 is very little evidence for female leaders in conflict resolution in the database, however. 293 Therefore, to expand on this modelling approach specifically for conflict resolution, a 294 logistic mixed effects model of Resolve conflict was fit (similarly to the Garfield, Syme, and 295 Hagen (2020) method with random intercepts for document authors nested within 296 cultures), but which excluded the leader sex term. This three-term model did show a 297 modest improvement in fit (using the cut off of $\triangle A I C<-2$, Burnham and Anderson 298 (2002)) over the intercept only model (including the random intercepts), $\triangle A I C=-2.33$.

299 Figure 3A plots the estimated marginal means of group context from the three-term logistic 300 mixed effects model of Resolve conflict and Table S2 reports results of an ANOVA test of the 301 fitted model. Continental region and subsistence type were not significant predictors of 302 Resolve conflict, however group context was a significant predictor ( $\mathrm{p}=0.02, \alpha=0.05)$. 303 Pairwise comparisons of estimated marginal means from levels of the group context 304 measure were compared using contrast analysis to identify the contexts which predicted evidence for Resolve conflict in the three-term model (Figure 3B). One pairwise comparison - kin group vs. political group (supracommunity) - produced a statistically significant mean difference (Tukey-adjusted $\mathrm{p}$-value $<0.05$ ) with kin groups producing the higher estimated marginal mean. 
Effects of group context on evidence for conflict resolution

A

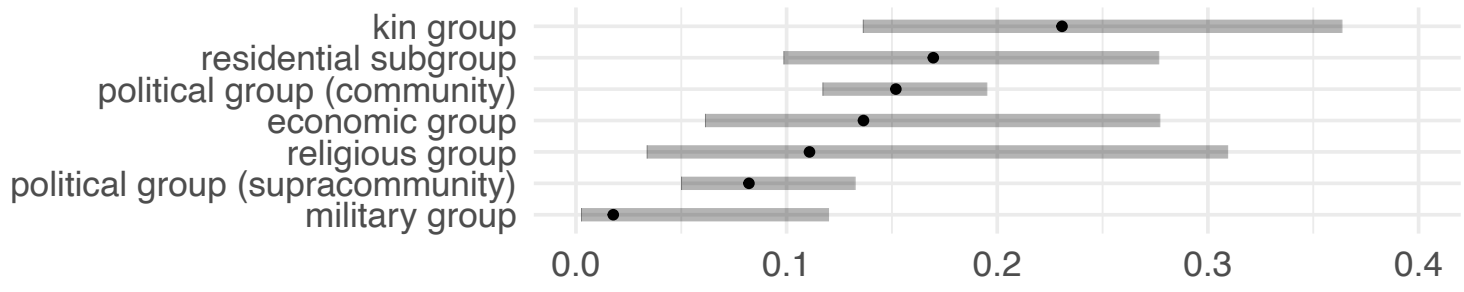

$\mathrm{B}$

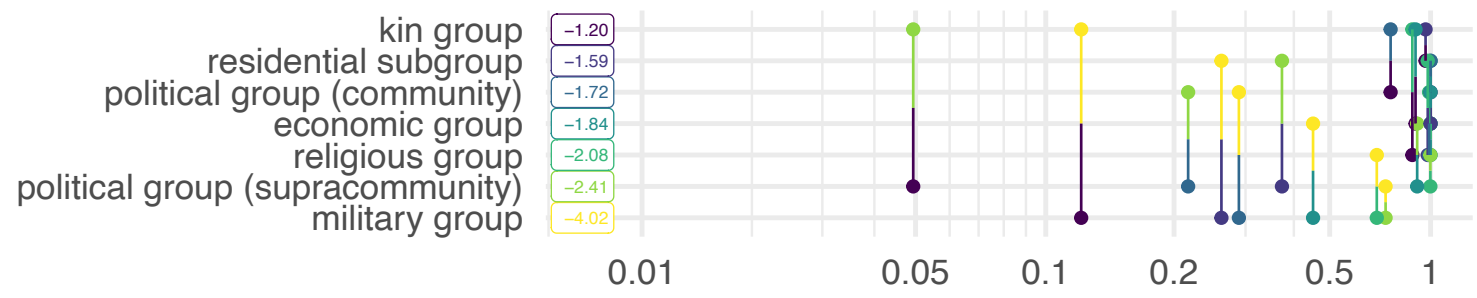

311

312 Figure 3: Effects of group context predicting evidence for conflict resolution (controlling for subsistence and region). A: Estimated marginal means of evidence for conflict resolution by group context from a three-term logistic mixed-effects regression model with random intercepts for author nested within culture. Values are on the response scale (probability). B: Contrast analysis plot from pairwise comparisons of estimated marginal means of levels of group context predictive evidence for conflict resolution (controlling for subsistence and

318 region). Values are Tukey-adjusted $p$ values.

\section{Text analysis of conflict resolution ethnography}

320 To empirically characterize the ethnographic record of conflict resolution, a documentterm matrix (DTM) was created of all "informative" words in the corpus of text records and the frequency with which they occurred in each text record (see Methods). The strongest positive predictors (terms) included, unsurprisingly, "dispute" and "quarrel," describing conflict scenarios. The next group of positive predictors included, "settle," "peace," and "mediator," describing resolution processes. Other notable positive predictors included, "decide," implicating decision making processes by leaders and a few terms specific to social contexts, such as "religious" and "political" supporting modelling results that social context is often mentioned in descriptions of conflict resolution. Several weak positive predictors were related to local and family contexts. Terms associated with more institutionalized and hierarchical leadership systems tended to be negative predictors. For example, "king," "leadership," and "chief" were negative predictors, suggesting the 
ethnographic record of leadership among kingdoms and more stratified social contexts are

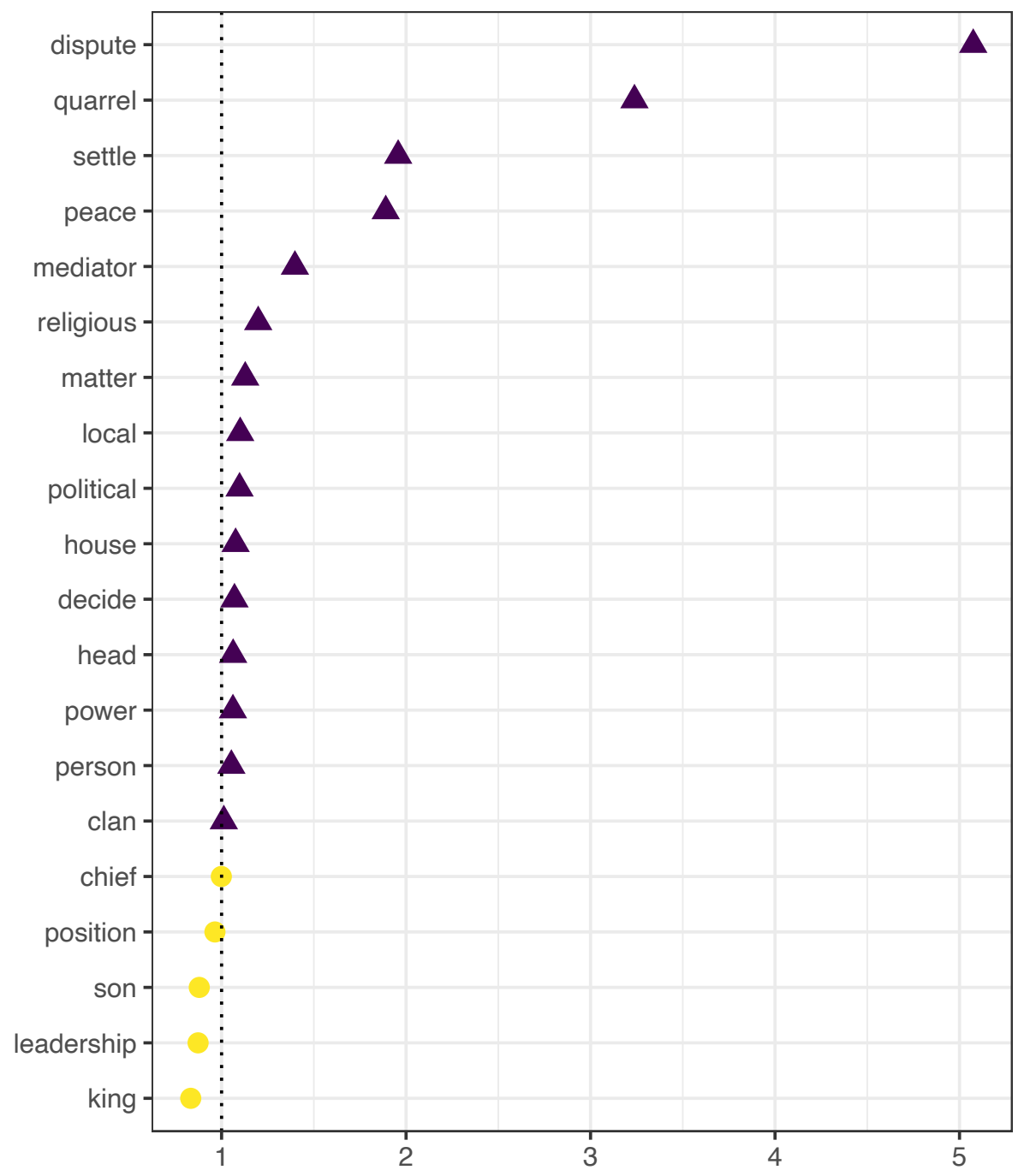

Figure 4: Non-zero coefficients of logistic elastic net text analysis regression model of evidence for Resolve conflict. Coefficients indicate the words whose frequencies in each text record best predicted evidence for Resolve conflict in each text record. Purple triangles indicate positive coefficients. Yellow circles indicate negative coefficients.

Identifying the traits commonly associated with conflict mediators has received substantial focus and is of broad multidisciplinary interest. The vast majority of studies on the behavioural and personality traits of conflict mediators, however, have relied on Western participants in either general or very specific postindustrial contexts (e.g., Moberg 1998; Sternberg and Soriano 1984; Corneliussen et al. 2017). Empirical support for such traits 
345 from a representative sample of human cultural diversity has not previously been available. Furthermore, most cross-cultural and ethnographic studies of conflict and conflict resolution have focused on inter-group conflicts (e.g., Ember and Ember 1994; Glowacki and Wrangham 2015; Ericksen and Horton 1992; Fry and Söderberg 2013), leaving a gap in the cross-cultural evidence for traits associated with conflict resolution within groups. The present study aimed to provide some insight towards filling these gaps.

\section{The correlates of conflict resolution}

352

353

354

355

356

357

358

359

360

361

362

363
Tehrani and Yamini (2020) provide a meta-analysis of psychological studies investigating associations between conflict resolution and personality traits (using the Five-Factor Personality Inventory) with five distinct conflict resolution styles: avoidance, compromising, integrating, obliging, and dominating. Relevant for the pattens observed here, they found 1) a positive relationship between extroversion and the dominating style of conflict resolution - where conflict mediators display a high concern for self, a low concern for others, and are selfish in influencing outcomes - and 2) agreeableness was negatively associated with dominating styles and positively associated with both an obliging style - where conflict mediators display a low concern for self, a high concern for others, and promote prosocial outcomes - and an integrating style - where conflict mediators display a high concern for self and others and promote mutually beneficial outcomes.

Results here on the importance of providing counsel and direction, interpersonal skills, and fairness among conflict mediators across cultures and contexts (Figure 1A) supports the relationships between agreeableness and the integrating and obliging styles of conflict resolution identified by Tehrani and Yamini (2020). Other group-service provision functions are more strongly related to evidence for conflict resolution in the ethnographic record than are many other theoretically important qualities. The association of providing counsel and direction in followers affairs, rather than evidence for coercion, fear, manipulation or physical formidability, suggests conflict resolution often involves leaders advising followers and providing input influencing outcomes rather than mandating specific outcomes. The ethnographic evidence suggests across cultures, conflict resolution is less likely to be associated with coercive dominance-based influence and more likely to be associated with freely conferred prestige-based influence (Redhead, Dhaliwal, and Cheng 2021). Leaders who resolve conflicts are also likely to perform other prosocial group functions including, group representation, counselling and advising followers, and providing protection and punishment services. Counselling followers in conflict is also key computational service and the connection between counselling and conflict resolution services lends some support the importance of mutually beneficial decision-making cognition as potential selective pressure in evolutionary models (Garfield, Hubbard, and Hagen 2019; Hagen and Garfield 2019).

Analyses here did not find an association of dominance-based leadership and conflict resolution. However, Garfield, Syme, and Hagen (2020) (using the same data analysed here) found that providing punishment services was the strongest positive predictor of leaders with coercive authority, a quality strongly implicated in the dominance style of leadership (Cheng 2019) and punishment services by leaders was moderately associated 
388

with conflict resolution in results here. Although qualities linked to dominance-based influence may not be strongly or directly associated with resolving conflicts in the ethnographic record, there is potentially an indirect connection between abilities to enforce punishment and resolve conflict, with dominance-based influence (Redhead, Dhaliwal, and Cheng 2021).

Empirical and ethnographic findings across diverse societies, including relatively egalitarian populations, suggests group representation often includes the development and maintenance of cooperative relationships and alliances (Boehm 1999; von Rueden et al. 2014; Bowser and Patton 2010). The importance of group representation in conflict resolution generally, lends some support Boehm's $(1982,329)$ speculation that, "when peace is actively created between fighting groups, it is likely that the same ideologies that so strongly support conflict resolution within the group are being applied in a similarly problem-solving fashion, but obviously in a between-group context."

It is worth note that the dimensions in the leadership data package capturing social capital; knowledge, experience, or decision-making capacities; or physical formidability were not identified as predictors of conflict resolution, as might be expected given related empirical and theoretical work (e.g., Glowacki and von Rueden 2015; Garfield, Hubbard, and Hagen 2019; Hagen and Garfield 2019) (though see Limitations). Interpersonal skills and fairness were the only leader qualities predictive of conflict resolution. This supports links between effective conflict resolution and moral evaluations of social justice, suggesting individuals who conform to and embody such traits are preferred as mediators. Effective mediators of conflict then are not necessarily leaders who can be conceptualized as particularly prestigious or dominant, but are more likely to be individuals who effectively identify overlapping interests between individuals with distinct priorities fairly, consistent with emerging views on leadership and followership focused on process and outcomes over inter-individual traits (Vollan et al. 2020; Wiessner 2019). Strong preferences for fairness potentially have deep evolutionary origins (Stavans and Baillargeon 2019; Nowak, Page, and Sigmund 2000; Haidt 2007). The importance of these leader qualities support an evolved psychology of procedural fairness and evaluations of welfare trade off ratios, or psychological preferences for leaders who will weight appropriately individual welfare in conflict resolution decision-making processes (Petersen et al. 2010; Sell, Tooby, and Cosmides 2009; Bøggild and Petersen 2016).

\section{Benefits associated with conflict resolution processes}

There were two benefits for followers associated with conflict resolution in the ethnographic record: Social services and Reduced risk of harm. These variables effectively identify the conflict resolution process itself. Two leader benefits, Increased social status and Social services were negative predictors of evidence for conflict resolution. This suggests that the well-known ethnographic descriptions of high status community leaders and the special services and privileges they receive are not often linked to descriptions of conflict resolution. Conflict resolution then might best be viewed as a prosocial service leaders or other individuals provide, for which they do not receive substantial direct benefits from. If so, this view then fails to support models reciprocal exchange of social service (e.g. conflict resolution) for social benefits or mutually beneficial outcomes (Price 
431 and Van Vugt 2014; Hagen and Garfield 2019; Garfield, Hubbard, and Hagen 2019). Alternatively, given the importance of conflict resolution in kin groups (see below) much of the ethnographic record of conflict resolution might be better understood as an investment in inclusive fitness or kin group welfare rather than a strategy for increased social mobility or personal gain (Hames 2015).

\section{The cultural ecology of conflict resolution}

437 Anthropologists have long debated the relative importance of concerted conflict management across populations with varying levels of "complexity," generally defined as more intensified subsistence economies and more elaborated and hierarchical sociopolitical structures (cf., Knauft et al. 1991; Wiessner 2016; Lee 1972). For example, Lee $(1972,182)$, downplaying within-group conflicts and conflict resolution among mobile

442 hunter-gatherers suggests,

"in contrast to agricultural and urban peoples, hunters have a great deal of latitude to vote with their feet, to walk out of an unpleasant situation. And they do so, not when the food supply is exhausted, but well before that point when only their patience is exhausted. This mobility has a profound ecological adaptive significance. Fear and avoidance of conflict has the effect of keeping people apart."

447 Demography is also implicated in shaping conflict rates and management and Lee $(2018,6)$ suggests, "living at very low densities, foragers had fewer things to fight over and, with little or no fixed property, could easily vote with their feet and disperse to diffuse conflict." Larger more densely populated, sedentary groups with more intensified economies and greater sociopolitical complexity are thought to have greater pressures of within and between group conflicts, and hence be equipped socially and politically with more elaborate conflict management processes. Despite any influence on conflicts due to mobility and demography, conflict resolution management and resolution systems are universal across human societies, including among mobile hunter-gatherers (see Boehm 2013). The "vote with their feet" hypothesis would suggest evidence for conflict resolution to be more frequent in the ethnographic record of agricultural and horticultural populations relative to more mobile hunter-gatherers or pastoralists. Exploratory analyses here, however, did not find variation in evidence for within or between group conflict resolution across cultures with different subsistence strategies (Figure 2A) and subsistence type did not predict evidence for conflict resolution (controlling for region and group context, Figure 3). Therefore, the "vote with their feet" hypothesis promoted by Lee and others and a cultural ecology of conflict resolution grounded in variation in subsistence strategies are not supported. One possible exception to this trend was the slightly greater frequency of evidence for between-group conflict resolution among pastoralists (Figure 2A). This could be due in part to pressures from shared resources related to livestockbased subsistence economies including access to water sources and grazing land and the diffuse and shifting nature of territoriality among pastoralists (Glowacki and Gonc 2013; Garfield, von Rueden, and Hagen 2019; Fratkin 2014).

Controlling for subsistence strategy and continental region, group context was a significant predictor of evidence for conflict resolution, with kin groups providing greater evidence for conflict resolution in contrast to higher-order political groups (Figure 3). Logistic regression identified a general trend of greater evidence for conflict resolution among 
474 more local and generalized groups (e.g., kin group, residential subgroups) compared to larger and more specialized groups (e.g., hierarchical political groups, military groups); which is supported by the text-analysis of conflict resolution ethnography (Figure 4). This trend suggests multiple, non-mutually exclusive interpretations. Leaders of higher order political groups may have other more demanding tasks than intervening in conflicts between group members and kin groups may experience greater demands or have a greater interest in resolving conflicts. Another set of interpretations would suggest, stronger influence of social institutions among populations with more developed political structures function to resolve conflicts with limited involvement of third-party individuals. Also, among more despotic and socially stratified societies, many potential conflicts may not manifest behaviourally given extreme power asymmetry between aggressor and victim. Future research should work to disentangle causal relationships between rates of conflicts by group context, social and political structures, and leadership functions.

Lastly, the ethnographic record of conflict resolution provides greater evidence for conflict resolution within-groups than between groups including across populations with variable subsistence strategies and across distinct social contexts (Figure 2 and see Limitations section). These results are consistent with recent work in anthropology emphasizing heterogeneity, variability, and social complexity among populations such as mobile huntergatherers, often characterized as "small-scale" (e.g., Hill et al. 2011; Bird et al. 2019; Singh and Glowacki 2021). Between-group conflicts, although relatively less represented, are consistently documented across all subsistence types including hunter-gatherers, providing additional evidence for the ubiquity of inter-group conflicts across the vast majority of human societies (Glowacki, Wilson, and Wrangham 2020; Hames 2019; Lopez 2016).

499 The current study has many important limitations. First, any ethnography-based analyses are necessarily restricted to the content ethnographers chose to record and publish. It is important to keep in mind information ethnographers were unaware of, disinterested in, nor permitted to research, constrains available information in any ethnographic document. For example, the biased evidence in favour of within-group conflicts could entirely be an artefact of ethnographer description. Furthermore, much (or most) ethnography is constructed from the perspective of the ethnographer (an emic view) and not necessarily from the perspective of members of the focal population (an etic view). Ethnographers'

508 Given the general content of text records in the leadership data package these results are
509 biased towards particular types of leaders, generally male political leaders. As previously perspectives on conflicts could be divergent from those of local community members. mentioned these data include very few text records pertaining to women as mediators of conflict. Results from this comparative, ethnography-based dataset, are also unable to provide direct comparison to related empirical and field-based research due to ecological framing differences, measurement and sampling differences, and the aforementioned limitations. 
515 All analyses conducted here are exploratory and correlational and do not suggest particular

516 leadership dimensions cause conflict resolution capacities or vice versa. Also, these data

517 cannot disentangle the degree to which leaders may be actively involved in, have a vested

518 interest in, and cause the conflicts in which they have been described in association with.

519 Given these limitations, these results can provide a comparative, empirical foundation for

520 future more detailed studies on the context of conflicts and their interactions with traits

521 associated with conflict mediators.

\section{Conclusion}

523 The ethnographic record provides evidence that conflict resolution by leaders often occurs

524 in the context of within-group conflicts across populations with variable subsistence

525 economies. Evidence for between-group conflict resolution, although more limited, is also

526 not biased towards any particular subsistence strategy. Culturally and economically

527 diverse populations likely face similar demands for conflict resolution and individual

528 leaders reliably emerge as conflict mediators across human societies.

529 Conflict resolution services are perhaps best viewed as part of larger leadership processes

530 aimed to promote within-group cohesion and strategic, beneficial between-group relations.

531 Variation in conflict resolution capacities are more likely to be associated with a wider

532 range of leadership functions, such as representing the group, providing counsel,

533 protecting group members, and punishing defectors, than particular personality traits or

534 individual qualities. The qualities of interpersonal skills and fairness, however are

535

536

537

538

539

540

541

542

543 potentially important for effective conflict resolution across diverse cultural and social contexts. These results suggest followers benefit primarily directly from conflict resolution processes, and there was not direct evidence that mediators of conflict receive reciprocal benefits such as special status or reciprocal services from followers. Although evidence for conflict resolution did not meaningfully vary across regions or by subsistence strategies, there was some evidence group context impacts demands of inter-individual conflict. More localized and smaller groups, such as kin groups more often feature leaders who actively resolve conflicts. Leaders within higher-order political groups are less likely to be actively involved in conflict resolution.

544 The ethnographic record is an indispensable tool for uncovering cultural diversity and universality. To better understand the potential for conflict emergence, scholars and managers should focus on micro-level group and social contexts, rather than macro-level group variation, such as subsistence economies or national characteristics. Groups with less hierarchical structure and greater face-to-face interaction may be predisposed to increased rates of within-group interpersonal conflicts. Individuals who generally provide group-beneficial services and have reputations for fairness and interpersonal skills may be best equipped to facilitate conflict resolution. Alternatively, increased internal organizational structure may also reduce the likelihood individual conflicts emerge and facilitate conflict mediation structurally. Theoretical models of conflict resolution can draw on the systematic results here in developing more robust, generalizable theories of conflict management. 
557 Luke Glowacki, Anthony Lopez, Kristen Syme, and Raymond Hames, as well as the

558 Evolutionary Human Sciences editor and two anonymous reviewers provided very useful

559 feedback on this manuscript. The data associated with this project were developed with

560 Edward Hagen and Kristen Syme and the framework draws on methods from Garfield,

561 Syme, and Hagen (2020).

\section{Financial Support}

563 This research was funded by National Science Foundation Division of Behavioral and 564 Cognitive Sciences award [1628509]. Zachary H. Garfield acknowledges IAST funding from 565 the French National Research Agency (ANR) under the Investments for the Future

566 (Investissements d'Avenir) program, grant ANR-17-EURE-0010.

\section{Conflicts of Interest declarations in manuscripts}

568 Author declares none.

\section{Research Transparency and Reproducibility}

570 The data associated with this project are available at

571 https://doi.org/10.5281/zenodo.2541999.

\section{References}

573 Alexander, Richard D. 1974. "The Evolution of Social Behavior." Annual Review of Ecology

574 and Systematics 5: 325-83.

575 https://doi.org/doi.org/10.1146/annurev.es.05.110174.001545.

576 Allee, Warder Clyde. 1951. Cooperation Among Animals: With Human Implications. Henry

577 Schuman.

578 Aureli, Filippo, Frans de Waal, and Frans B. M. Waal. 2000. Natural Conflict Resolution.

579 University of California Press.

580 Bates, Douglas, Martin Mächler, Ben Bolker, and Steve Walker. 2015. "Fitting Linear Mixed-

581 Effects Models Using lme4.” Journal of Statistical Software 67 (1): 1-48.

582 https://doi.org/10.18637/jss.v067.i01.

583 Bird, Douglas W., Rebecca Bliege-Bird, Brian F. Codding, and David W. Zeanah. 2019.

584 "Variability in the Organization and Size of Hunter-Gatherer Groups: Foragers Do Not Live

585 in Small-Scale Societies." Journal of Human Evolution 131: 96-108.

586 https://doi.org/10.1016/j.jhevol.2019.03.005.

587 Boehm, C. 1999. Hierarchy in the Forest: The Evolution of Egalitarian Behavior. Harvard

588 University Press Cambridge, MA. 
589

590

591

592

593

594

595

596

597

598

599

600

601

602

603

604

605

606

607

608

609

610

611

612

613

614

615

616

617

618

619

620

621

622

623

624

-_- 2008. "Purposive Social Selection and the Evolution of Human Altruism." CrossCultural Research 42: 319-54.

Boehm, Christopher. 1982. "The Evolutionary Development of Morality as an Effect of Dominance Behavior and Conflict Interference." JSOCBS Journal of Social and Biological Structures 5 (4): 413-21.

-_- 2013. "The Biocultural Evolution of Conflict Resolution Between Groups." In War, Peace, and Human Nature: The Convergence of Evolutionary and Cultural Views, edited by D. P. Fry, 315-40. Oxford: Oxford University Press.

Bowser, Brenda, and John Patton. 2010. "Women's Leadership: Political Alliance, Economic Resources, and Reproductive Success in the Ecuadorian Amazon." In The Evolution of Leadership: Transitions in Decision Making from Small-Scale to Middle-Range Societies, edited by Kevin J. Vaughn, Jelmer W. Eerkins, and John Kanter, 51-71. Sante Fe: SAR.

Brown, Donald E. 1991. Human Universals. McGraw-Hill New York.

Burnham, Kenneth P., and David R. Anderson, eds. 2002. Model Selection and Multimodel Inference: A Practical Information-Theoretic Approach. Springer New York.

https://doi.org/10.1007/b97636.

Bøggild, Troels, and Michael Bang Petersen. 2016. "The Evolved Functions of Procedural Fairness: An Adaptation for Politics." In The Evolution of Morality, edited by Todd K. Shackelford and Ronald D. Hansen, 247-76. Springer.

Chapman, Colin A., and Kim Valenta. 2015. "Costs and Benefits of Group Living Are Neither Simple nor Linear." Proceedings of the National Academy of Sciences of the United States of America 112 (48): 14751-2. https://doi.org/10.1073/pnas.1519760112.

Cheng, Joey T. 2019. "Dominance, Prestige, and the Role of Leveling in Human Social Hierarchy and Equality." Current Opinion in Psychology, October. https://doi.org/10.1016/j.copsyc.2019.10.004.

Corneliussen, Jesper G., Gloria R. Leon, Anders Kjærgaard, Birgit A. Fink, and Noah C. Venables. 2017. "Individual Traits, Personal Values, and Conflict Resolution in an Isolated, Confined, Extreme Environment." Aerospace Medicine and Human Performance 88 (6): 53543. https://doi.org/10.3357/AMHP.4785.2017.

Ember, Carol R., and Melvin Ember. 1994. "War, Socialization, and Interpersonal Violence: A Cross-Cultural Study." Journal of Conflict Resolution 38 (4): 620-46. https://doi.org/10.1177/0022002794038004002.

Ericksen, Karen Paige, and Heather Horton. 1992. "Blood Feuds": Cross-Cultural Variations in Kin Group Vengeance." Cross-Cultural Research 26: 57-85.

Fratkin, Elliot. 2014. “Ethiopia's Pastoralist Policies: Development, Displacement and Resettlement." Nomadic Peoples 18: 94-114. 
625 Friedman, Jerome, Trevor Hastie, and Robert Tibshirani. 2010. "Regularization Paths for

626 Generalized Linear Models via Coordinate Descent." Journal of Statistical Software 33 (1): 1.

627 Fry, D. P., and P. Söderberg. 2013. "Lethal Aggression in Mobile Forager Bands and

628 Implications for the Origins of War." Science (New York, N Y) 341: 270-3.

629 Garfield, Zachary H., and Edward Hagen. 2019. "z4ch4ry/leadershipdata: Garfield,

630 Hubbard, \& Hagen, 2019, Evolutionary models of leadership: Tests and synthesis." Zenodo.

631 https://doi.org/10.5281/zenodo.4018788.

632 Garfield, Zachary H., H. Hubbard Robert, and Edward H. Hagen. 2019. “Evolutionary Models 633 of Leadership: Tests and Synthesis." Human Nature 30 (1): 23-58.

634 https://doi.org/10.1007/s12110-019-09338-4.

635 Garfield, Zachary H., Kristen L. Syme, and Edward H. Hagen. 2020. "Universal and Variable 636 Leadership Dimensions Across Human Societies." Evolution and Human Behavior 41 (5):

637 397-414. https://doi.org/10.1016/j.evolhumbehav.2020.07.012.

638 Garfield, Zachary H., Christopher von Rueden, and Edward H. Hagen. 2019. "The

639 Evolutionary Anthropology of Political Leadership." The Leadership Quarterly 30 (1): 59-

640 80. https://doi.org/10.1016/j.leaqua.2018.09.001.

641 Gavrilets, Sergey, and Laura Fortunato. 2014. "A Solution to the Collective Action Problem

642 in Between-Group Conflict with Within-Group Inequality." Nature Communications 5: 3526.

643 Glowacki, Luke, and Katja Gonc. 2013. "Customary Institutions and Traditions in Pastoralist 644 Societies: Neglected Potential for Conflict Resolution." Conflict Trends 2013 (1): 26-32.

645 Glowacki, Luke, and Christopher von Rueden. 2015. “Leadership Solves Collective Action 646 Problems in Small-Scale Societies." Philos Trans R Soc Lond B Biol Sci 370 (20150010).

647 https://doi.org/10.1098/rstb.2015.0010.

648 Glowacki, Luke, Michael L. Wilson, and Richard W. Wrangham. 2017. "The evolutionary anthropology of war." Journal of Economic Behavior and Organization.

651 - - 2020. "The Evolutionary Anthropology of War." Journal of Economic Behavior \& 652 Organization 178 (October): 963-82. https://doi.org/10.1016/j.jebo.2017.09.014.

653 Glowacki, Luke, and Richard Wrangham. 2015. "Warfare and Reproductive Success in a 654 Tribal Population." Proc Natl Acad Sci U S A 112 (January): 348-53.

655 https://doi.org/10.1073/pnas.1412287112.

656 Gómez, José María, Miguel Verdú, Adela González-Megías, and Marcos Méndez. 2016. “The 657 Phylogenetic Roots of Human Lethal Violence." Nature 538 (7624): 233-37.

658 https://doi.org/10.1038/nature19758. 
659 Hagen, Edward H, and Zachary H. Garfield. 2019. "Leadership and Prestige, Mothering, 660 Sexual Selection, and Encephalization: The Computational Services Model." Preprint. Open 661 Science Framework. https://doi.org/10.31219/osf.io/9bcdk.

662 Haidt, Jonathan. 2007. "The New Synthesis in Moral Psychology." Science 316 (May): 998663 1002. https://doi.org/10.1126/science.1137651.

664 Hames, Raymond. 2015. "Kin Selection.” In The Handbook of Evolutionary Psychology, 665 edited by David Buss, Second edition, 2:505-23. Hoboken, New Jersey: John Wiley \& Sons, 666 Inc.

667 - - 2019. "Pacifying Hunter-Gatherers." Human Nature 30 (2): 155-75.

668 https://doi.org/10.1007/s12110-019-09340-w.

669 Hill, Kim R., Robert S. Walker, Miran Bozicević, James Eder, Thomas Headland, Barry

670 Hewlett, A. Magdalena Hurtado, Frank Marlowe, Polly Wiessner, and Brian Wood. 2011.

671 "Co-Residence Patterns in Hunter-Gatherer Societies Show Unique Human Social

672 Structure." Science 331 (March): 1286-9. https://doi.org/10.1126/science.1199071.

673 Hrdy, Sarah Blaffer. 2006. "Evolutionary Context of Human Development. The Cooperative 674 Breeding Model." Family Relationships, 39.

675 Jensen, Keith. 2010. "Punishment and Spite, the Dark Side of Cooperation." Philosophical 676 Transactions of the Royal Society B: Biological Sciences 365 (1553): 2635-50.

677 https://doi.org/10.1098/rstb.2010.0146.

678 Knauft, Bruce M., Thomas S. Abler, Laura Betzig, Christopher Boehm, Robert Knox Dentan, 679 Thomas M. Kiefer, Keith F. Otterbein, John Paddock, and Lars Rodseth. 1991. "Violence and 680 Sociality in Human Evolution [and Comments and Replies]." Current Anthropology 32 (4): 681 391-428. https://doi.org/10.1086/203975.

682 Lee, Richard B. 1972. "Work Effort, Group Structure, and Land Use in Contemporary 683 Hunter-Gatherers." In Men, Settlement, and Urbanism, edited by P. J. Ucko, R. Tringham, and 684 G. W. Dimbleby, 177-85. Duckworth.

685 - - 2018. "Hunter-Gatherers and Human Evolution."

686 Lopez, Anthony. 2020. "Evolutionary Psychology and Warfare." In The Sage Handbook of 687 Evolutionary Psychology: Applications of Evolutionary Psychology, edited by Todd K.

688 Shackelford.

689 Lopez, Anthony C. 2016. "The Evolution of War: Theory and Controversy." Int Theory 690 International Theory 8: 97-139.

691 Mesterton-Gibbons, Michael, and Lee Alan Dugatkin. 1995. "Toward a Theory of Dominance 692 Hierarchies: Effects of Assessment, Group Size, and Variation in Fighting Ability."

693 Behavioral Ecology 6: 416-23. 
694 Mesterton-Gibbons, Mike, Sergey Gavrilets, Janko Gravner, and Erol Akçay. 2011. "Models of Coalition or Alliance Formation." Journal of Theoretical Biology 274 (1): 187-204.

696 https://doi.org/10.1016/j.jtbi.2010.12.031.

697 Moberg, Philip J. 1998. "Predicting Conflict Strategy with Personality Traits: Incremental 698 Validity and the Five Factor Model." International Journal of Conflict Management 9 (3):

699 258-85. https://doi.org/10.1108/eb022812.

700 Naroll, Raoul. 1967. “The Proposed HRAF Probability Sample.” Cross-Cultural Research 2: $701 \quad 70-80$.

702 Nowak, M. A., K. M. Page, and K. Sigmund. 2000. "Fairness Versus Reason in the Ultimatum 703 Game." Science 289 (September): 1773-5.

704 Parker, G. A. 2006. "Sexual Conflict over Mating and Fertilization: An Overview."

705 Philosophical Transactions of the Royal Society of London B: Biological Sciences 361 (1466):

706 235-59. https://doi.org/10.1098/rstb.2005.1785.

707 Parker, Geoff A., Nick J. Royle, and Ian R. Hartley. 2002. "Intrafamilial Conflict and Parental 708 Investment: A Synthesis.” Philosophical Transactions: Biological Sciences 357 (1419): 295709307.

710 Petersen, Michael Bang, Aaron Sell, John Tooby, and Leda Cosmides. 2010. "Evolutionary 711 Psychology and Criminal Justice: A Recalibrational Theory of Punishment and 712 Reconciliation." In Human Morality \& Sociality: Evolutionary \& Comparative Perspectives, 713 edited by H Høegh-Olesen, 72-131. New York: Palgrave Macmillan.

714 Pospisil, Leopold J. 1963. Kapauku Papuan Economy. New Haven: Dept. of Anthropology, 715 Yale University.

716 Price, Michael E., and Mark Van Vugt. 2014. “The Evolution of Leader-Follower Reciprocity:

717 The Theory of Service-for-Prestige." Frontiers in Human Neuroscience 8 (363): 1-17.

718 https://doi.org/10.3389/fnhum.2014.00363.

719 Raihani, Nichola, and Eleanor A. Power. 2021. "No Good Deed Goes Unpunished: The Social 720 Costs of Prosocial Behaviour,” May. https://doi.org/10.31234/osf.io/ebfrg.

721 Redhead, Daniel, Nathan Dhaliwal, and Joey T. Cheng. 2021. "Taking Charge and Stepping

722 in: Individuals Who Punish Are Rewarded with Prestige and Dominance." Social and

723 Personality Psychology Compass 15 (2): e12581. https://doi.org/10.1111/spc3.12581.

724 Redhead, Daniel, and Christopher Von Rueden. 2021. “Coalitions and Conflict: A

725 Longitudinal Analysis of Men's Politics.” Evolutionary Human Sciences, 1-28.

726 https://doi.org/10.1017/ehs.2021.26.

727 Richerson, Peter, Ryan Baldini, Adrian V. Bell, Kathryn Demps, Karl Frost, Vicken Hillis, 728 Sarah Mathew, Emily K. Newton, Nicole Naar, and Lesley Newson. 2016. "Cultural Group 729 Selection Plays an Essential Role in Explaining Human Cooperation: A Sketch of the

730 Evidence." Behavioral and Brain Sciences 39. 
731 Roscoe, P. 2009. "Social Signaling and the Organization of Small-Scale Society: The Case of Contact-Era New Guinea.” Journal of Archaeological Method and Theory 16: 69-116.

733 Ross, Marc Howard. 1983. "Political Decision Making and Conflict: Additional Cross-

734 Cultural Codes and Scales.” Ethnology 22 (April): 169. https://doi.org/10.2307/3773578.

735 Santos, Miguel dos, and Stuart A. West. 2018. "The Coevolution of Cooperation and

736 Cognition in Humans." Proc. R. Soc. B 285 (1879): 20180723.

737 https://doi.org/10.1098/rspb.2018.0723.

738 Sell, Aaron, John Tooby, and Leda Cosmides. 2009. "Formidability and the Logic of Human Anger." Proceedings of the National Academy of Sciences 106 (35): 15073.

740 https://doi.org/10.1073/pnas.0904312106.

741 Singh, Manvir, and Luke Glowacki. 2021. "Human Social Organization During the Late 742 Pleistocene: Beyond the Nomadic-Egalitarian Model," March.

743 https://doi.org/10.32942/osf.io/vusye.

744 Sluka, Jeffrey A. 1992. “The Anthropology of Conflict.” In The Paths to Domination,

745 Resistance, and Terror, 18-36.

746 Smith, Eric Alden. 1985. "Inuit Foraging Groups: Some Simple Models Incorporating

747 Conflicts of Interest, Relatedness, and Central-Place Sharing." Ethology and Sociobiology 6:

$748 \quad 27-47$.

749 Smith, Jennifer E., Sergey Gavrilets, Monique Borgerhoff Mulder, Paul L. Hooper, Claire El Mouden, Daniel Nettle, Christoph Hauert, et al. 2016. "Leadership in Mammalian Societies:

751

752 Emergence, Distribution, Power, and Payoff.” Trends Ecol Evol 31 (January): 54-66. https://doi.org/10.1016/j.tree.2015.09.013.

753 Smith, Jennifer E., Eli M. Swanson, Daphna Reed, and Kay E. Holekamp. 2012. "Evolution of Cooperation Among Mammalian Carnivores and Its Relevance to Hominin Evolution."

755 Current Anthropology 53 (S6): S436-S452.

756 Stavans, Maayan, and Renée Baillargeon. 2019. "Infants Expect Leaders to Right Wrongs."

757 Proceedings of the National Academy of Sciences, July, 201820091.

758 https://doi.org/10.1073/pnas.1820091116.

759 Sternberg, Robert J., and Lawrence J. Soriano. 1984. "Styles of Conflict Resolution." Journal

760 of Personality and Social Psychology 47 (1): 115-26. https://doi.org/10.1037/0022-

7613514.47 .1 .115$.

762 Tehrani, Hossein Dabiriyan, and Sara Yamini. 2020. "Personality Traits and Conflict 763 Resolution Styles: A Meta-Analysis.” Personality and Individual Differences 157 (April):

764 109794. https://doi.org/10.1016/j.paid.2019.109794.

765 Vollan, Björn, Esther Blanco, Ivo Steimanis, Fabian Petutschnig, and Sebastian Prediger. 766 2020. "Procedural Fairness and Nepotism Among Local Traditional and Democratic 
767 Leaders in Rural Namibia." Science Advances 6 (15): eaay7651.

768 https://doi.org/10.1126/sciadv.aay7651.

769 von Rueden, Christopher, Michael Gurven, Hillard Kaplan, and Jonathan Stieglitz. 2014.

770 "Leadership in an Egalitarian Society." Hum Nat 25 (4): 538-66.

771 https://doi.org/10.1007/s12110-014-9213-4.

772 von Rueden, Christopher, and Mark van Vugt. 2015. "Leadership in Small-Scale Societies:

773 Some Implications for Theory, Research, and Practice." The Leadership Quarterly 26

774 (December): 978-90. https://doi.org/10.1016/j.leaqua.2015.10.004.

775 Wiessner, Polly. 2019. "Collective Action for War and for Peace: A Case Study Among the 776 Enga of Papua New Guinea." Current Anthropology 60 (2): 224-44.

777 https://doi.org/10.1086/702414.

778 - - 2016. "The Rift Between Science and Humanism: What's Data Got to Do with It?"

779 Current Anthropology 57 (S13): S154-S166. https://doi.org/10.1086/686017.

780 Willems, Erik P., and Carel P. van Schaik. 2017. "The Social Organization of Homo Ergaster:

781 Inferences from Anti-Predator Responses in Extant Primates." Journal of Human Evolution

782109 (August): 11-21. https://doi.org/10.1016/j.jhevol.2017.05.003.

783 Wilson, Don E., and DeeAnn M. Reeder. 2005. Mammal Species of the World: A Taxonomic 784 and Geographic Reference. JHU Press. 